\title{
Flu Pandemic Preparedness: Detection of the Potentially Pandemic G4 EA Avian-Like H1N1 Strains Using QIAstat-Dx Respiratory SARS-CoV-2 Panel
}

\section{Luis Penarrubia}

QIAGEN Inc, Barcelona https://orcid.org/0000-0001-6024-1832

Sonia N. Rao

QIAGEN Inc, Germantown https://orcid.org/0000-0002-3850-6395

Roberto Porco

QIAGEN Inc, Barcelona https://orcid.org/0000-0002-1108-5412

Marta Varo

QIAGEN Inc, Barcelona https://orcid.org/0000-0001-5149-9655

\section{Patricia Muñoz-Torrero}

QIAGEN Inc, Barcelona

Josep Pareja ( $\nabla$ josep.pareja@qiagen.com )

QIAGEN Inc, Barcelona https://orcid.org/0000-0001-9075-3417

Marta López-Fontanals

QIAGEN Inc, Barcelona

\section{Davide Manissero}

QIAGEN Manchester Ltd https://orcid.org/0000-0002-8887-8795

\section{Research Article}

Keywords: Influenza A, Swine Flu, Multiplex PCR

Posted Date: August 2nd, 2020

DOl: https://doi.org/10.21203/rs.3.rs-50897/v1

License: @ (i) This work is licensed under a Creative Commons Attribution 4.0 International License. Read Full License 


\section{Abstract}

A recent publication by Sun $\mathrm{H}$. and colleagues in the Proceedings of the National Academy of Science (PNAS) has drown global attention to the predominant genotype "G4" Eurasian (EA) avian-like H1N1 Influenza A virus that has been spreading among pigs in China since 2016, which is predicted to have a significant pandemic potential. Since pigs are hosts for the generation of pandemic Influenza A viruses (IAVs), surveillance and preparedness are critical to prevent pandemics. In this regards, one distinguishing feature of the QIAstat-Dx ${ }^{\circledR}$ Respiratory SARS-CoV-2 panel is the double target approach Influenza A detection of seasonal strains affecting humans, by amplification of a generic Influenza A assay plus one of the specific assays discriminating $\mathrm{H} 3, \mathrm{H} 1$ and $\mathrm{H} 1 \mathrm{~N} 1$ pdm09 subtypes. The generic Influenza A assay is designed to amplify any IAV, a key feature for preparedness. Here we report an initial in-silico analysis that predicts that the G4 EA avian-like H1N1 strains tested in the QIAstat-Dx ${ }^{\circledR}$ Respiratory SARS-CoV-2 Panel would be detected yielding a positive result for the generic Influenza $A$ assay and negative results for the seasonal $\mathrm{H} 3, \mathrm{H} 1$ and $\mathrm{H} 1 \mathrm{~N} 1 \mathrm{pdm} 09$ assays. This prediction was confirmed in-vitro using dsDNA fragments mixed to mimic the genomes of the different reported G4 EA IAV strains. In conclusion, the QIAstat-Dx ${ }^{\circledR}$ Respiratory SARS-CoV-2 Panel could be a useful tool to differentially diagnose zoonotic strains from the seasonal Influenza A strains commonly affecting humans.

\section{Introduction}

One important public health lesson learned from the Severe Acute Respiratory Syndrome Coronavirus 2 (SARS-CoV-2) pandemic has been the importance of time to action and preparedness for detection of novel viruses with pandemic potential. A recent publication in the Proceedings of the National Academy of Science (PNAS) by Sun H. and colleagues described the predominant genotype "G4" Eurasian (EA) avian-like H1N1 virus that has been spreading among pigs in China since 2016, which has H1N1 pandemic 2009 (H1N1pdm09) and triple-reassortant (TR)-derived internal genes (1). It is well established that efficient human-to-human transmission is a critical feature of pandemic influenza viruses $(2,3)$. The authors determined that the emergent novel G4 reassortment EA H1N1 virus has high infectivity and transmissibility potential via direct contact and respiratory droplets amongst ferrets, suggesting ability to infect humans as the previous pandemic $\mathrm{H} 1$ in 2009 (4,5). Additionally, the reported serological surveillance amongst swine workers with occupational exposure showed $10.4 \%$ were positive for G4 swine EA H1N1 with younger patients 18-35 years old with higher rates of seropositivity. These data caught the attention of the US Center for Disease Control and Prevention which has already determined that their current Influenza Virus Real-time RT-PCR Influenza A (H1/H3/H1N1pdm09) Subtyping panel would detect this swine influenza virus (SIV) (6). This would yield a positive result for the subtype H1N1, which would indicate that the H1N1 subtype assay shows cross-reactivity to swine variants of the H1 genotype. 
The overlap of viral etiologies of Influenza Like Illness (ILI) during the influenza season has increased the attention to syndromic diagnostics with multiplex capability, as well as the need to increase ability to detect and distinguish new variants. The QIAstat-Dx ${ }^{\circledR}$ Respiratory SARS-CoV-2 Panel offers a multiplex real time RT-PCR platform with 22 targets (CE-IVD) or 21 targets (FDA EUA) as one of the currently available respiratory syndromic solutions (7).

One distinguishing feature of the QIAstat-Dx ${ }^{\circledR}$ Respiratory SARS-CoV-2 panel is the double target approach to Influenza A detection. Specifically, this detection is based on a generic assay designed to detect any Influenza A strain, as well as 3 specific assays to discriminate between the three main Influenza A Virus (IAV) subtypes infecting humans: seasonal IAV H1, seasonal IAV H3, and IAV H1N1pdm09. The dual target approach enhances the detection of the IAV genotypes affecting humans, while allowing the detection of novel strains by detecting Influenza A targets without subtype (ruling out $\mathrm{H} 1, \mathrm{H} 3$ and $\mathrm{H} 1 \mathrm{~N} 1 \mathrm{pdm09}$ ). The mentioned generic Influenza A design targets the Matrix gene as the one with highest homology among IAV.

After performing an in-silico analysis of the G4 EA H1N1 strains defined in Sun H. et al. (1) against the 4 Influenza A assays included in the QIAstat-Dx ${ }^{\circledR}$ Respiratory SARS-CoV-2 Panel, we generated the hypothesis that upon testing with the QIAstat-Dx ${ }^{\circledR}$ Respiratory SARS-CoV-2 Panel, the G4 EA H1N1pdm09like lineage would be detected as generic Influenza A positive with no subtype discrimination given the specificity of the human $\mathrm{H} 1$ and $\mathrm{H} 1 \mathrm{~N} 1 \mathrm{pdm} 09$ subtype assays on the panel. We sought to test this hypothesis in-vitro to confirm that the QIAstat-Dx ${ }^{\circledR}$ Respiratory SARS-CoV-2 Panel would be able to detect this new $\mathrm{G} 4$ swine EA H1N1 strain providing the expected level of specificity.

\section{Results}

Bioinformatic analysis showed high homology between the generic Influenza A assay (no subtype) included in the QIAstat-Dx ${ }^{\circledR}$ Respiratory SARS-CoV-2 Panel and all screened 29 G4 strains. Mismatches were low in frequency and located in no-critical positions in the oligonucleotide sequences, rendering a positive amplification prediction by the generic Influenza A assay when testing the described any of the described G4 swine strains.

On the contrary, detection by the Influenza A H1 subtypes assays ( $\mathrm{H} 1$ and $\mathrm{H} 1 \mathrm{~N} 1 \mathrm{pdm} 09)$ was as predicted negative. Several mismatches were found in both seasonal H1 and H1N1pdm09 RT-qPCR assays, predicting no amplification by RT-PCR.

Further laboratory testing of simulated G4 strains corroborated the in-silico prediction (Table 1). All 13 sample combinations covering the complete genetic variability observed among the $29 \mathrm{G} 4$ strains resulted in Influenza A positive with no subtype discrimination. None of the subtype assays presented a cross-reaction with this new G4 genetic cluster. Those results demonstrate the good specificity of the QIAstat-Dx ${ }^{\circledR}$ Respiratory SARS-CoV-2 Panel with the ability to discriminate only human-specific IAV H1 
strains. The presence of EA capsid genes (HA and NA genes) of the swine G4 lineage (1) facilitate the discrimination from human $\mathrm{H} 1$ or $\mathrm{H} 1 \mathrm{~N} 1 \mathrm{pdm} 09$ subtypes.

\section{Discussion}

Although there are currently no reports of the Influenza G4 EA H1N1pdm09-like viruses spreading from person-to-person, it is important to remain vigilant of potentially infective Influenza strains from other species and be prepared to take action in the event of a public health threat $(2,4,5)$. To cover the possibility of a spillover from other species, the overall detection strategy of Influenza A in the QIAstatDx ${ }^{\circledR}$ Respiratory SARS-CoV-2 Panel is to specifically detect and differentiate the human seasonal $\mathrm{H} 1$, the human seasonal $\mathrm{H} 3$ and the H1N1pdm09 subtypes while providing a generic Influenza A-no subtype assay. This detection strategy using a generic Influenza A assay fulfils the double function of confirmation of the human-specific subtypes detection while also allowing the potential detection of nonhuman IAVs. In this regards, the generic Influenza A assay in the QIAstat-Dx ${ }^{\circledR}$ Respiratory SARS-CoV-2 Panel is predicted to detect the recorded zoonotic variants such as avian IAVs subtypes A(H5N1), $A(H 7 N 9)$, and $A(H 9 N 2)$ and swine IAVs subtypes $A(H 1 N 1), A(H 1 N 2)$ and $A(H 3 N 2)(8)$.

Here we confirm experimentally that the newly emergent swine Influenza A G4 EA H1N1pdm09-like viruses reported by Sun $\mathrm{H}$. and colleagues $\left(1\right.$ ) would be detected by the QIAstat-Dx ${ }^{\circledR}$ Respiratory SARSCoV-2 Panel yielding a positive generic Influenza A result and negative result for specific human $\mathrm{H} 3, \mathrm{H} 1$ and $\mathrm{H} 1 \mathrm{~N} 1 \mathrm{pdm} 09$ subtypes. This contrasts with the report by the $\mathrm{CDC}$ of the detection of this potentially pandemic emergent swine $\mathrm{G} 4$ strain as seasonal H1N1 positive result using the Influenza Virus Real-time RT-PCR Influenza A (H1/H3/H1N1pdm09) Subtyping panel (6).

The results we report supports the overall Influenza A detection strategy of the QIAstat-Dx ${ }^{\circledR}$ Respiratory SARS-CoV-2 Panel allowing human-specific Influenza A subtype detection as well as non-human IAVs. In conclusion, in the event that the G4 swine EA IAV adaptation causes human-to-human transmission, the QIAstat-Dx ${ }^{\circledR}$ Respiratory SARS-CoV-2 Panel could be a useful tool to differentially diagnose zoonotic strains from the seasonal IAVs commonly affecting humans.

\section{Materials And Methods}

A thorough bioinformatic analysis of the possible amplification of the G4 swine EA H1N1 pdm09-like lineage was performed. The entire set of Genbank sequence accessions of the $29 \mathrm{G} 4$ strains described in Sun $\mathrm{H}$. et al. (1) were collected including the eight fragments of every screened genome. An alignment between the different sections of the viral genomes and the Influenza A assays targeted by the QIAstatDx ${ }^{\circledR}$ Respiratory SARS-CoV-2 panel was carried out using ClustalW algorithm implemented in Geneious software v.10.2.6 (http://www.geneious.com), with a gap open and extent cost of 5 and 3 respectively. The output alignment was curated manually. 
A specificity assessment on the criticality of the mismatches between the viral sequences and the oligonucleotide sequences was made to establish a prediction on the potential amplification of the targeted regions of the G4 EA H1N1pdm09-like viral genomes. Every screened primer set (general Influenza A detection; and $\mathrm{H} 1, \mathrm{H} 3$ and $\mathrm{H} 1 \mathrm{~N} 1 \mathrm{pdm} 09$ subtypes) was considered unspecific for G4 EA lineage if: 1) three or more mismatches were found among any oligonucleotide sequence and the strain gene sequences or 2) variations were placed in the three last nucleotides of the 3 '-end or in the 5 '-end of the probe affecting the PCR amplification $(9,10,11)$. This approach has been used previously to characterize inclusivity of specific RT-qPCR assays for viral detection (12).

In addition, laboratory testing was performed to confirm in-silico prediction. A total of $22 \mathrm{gBlocks}$ (dsDNA fragments of $500 \mathrm{bp}$ size) were designed to cover all the genetic variability observed in the sequence alignments among the 29 G4 EA strains described in Sun H. et al. (1), corresponding to the different Influenza A genome regions targeted by the QIAstat-Dx ${ }^{\circledR}$ Respiratory SARS-CoV-2 Panel. Those 22 gBlocks were mixed accordingly in 13 unique combinations ( $1 \mathrm{E}+05$ copies $/ \mathrm{mL}$ concentration) in order to simulate all the genetic variability of the viral genomic regions targeted by the QIAstat-Dx ${ }^{\circledR}$ Respiratory SARS-CoV-2 Panel. Finally, two analytical samples were also included in the analysis as controls corresponding to a known seasonal $\mathrm{H} 1$ and $\mathrm{H} 1 \mathrm{~N} 1$ pdm09 human strains (Table 1).

\section{Declarations}

\section{Acknowledgments}

We thank Sun $\mathrm{H}$ et al. (2020) for their comprehensive and significant work. We also thank the QIAstat-Dx team for their support in their corresponding roles.

Competing Interest Statement: LP, SNR, RP, MV, PMT, JP, MLF and DM are QIAGEN employees.

\section{References}

1. Sun, et al. Prevalent Eurasian avian-like H1N1 swine influenza virus with 2009 pandemic viral genes facilitating human infection. Proc. Natl. Acad. Sci. U.S.A.117 (29), 17204-17210 (2020). https://doi.org/10.1073/pnas.1921186117.

2. Krumbholz, et al. Origin of the European avian-like swine influenza viruses. J. Gen. Virol. 95, 23722376 (2014). https://doi.org/10.1099/vir.0.068569-0.

3. K. Anderson, et al. Phylogeny-Based Global Nomenclature System and Automated Annotation Tool for H1 Hemagglutinin Genes from Swine Influenza A Viruses. mSphere. 1 e00275-16 (2016). https://doi.org/10.1128/mSphere.00275-16.

4. J. Watson, et al. Molecular Epidemiology and evolution of influenza viruses circulating within European swine between 2009 and 2013. J. Virol. 89, 9920-9931 (2015). https://doi.org/10.1128/jvi.00840-15. 
5. Henritzi, et al. Surveillance of European domestic pig populations identifies an emerging reservoir of potentially zoonotic Swine influenza A viruses. Cell Host Microbe 28, 1-14 (2020). https://doi.org/10.1128/jvi.00840-15.

6. Centers for Disease Control and Prevention, National Center for Immunization and Respiratory Disease (NCIRD). Available at: https://www.cdc.gov/flu/spotlights/2019-2020/cdc-prepare-swineflu.html. Accessed: 27 Jul 2020.

7. QIAstat-Dx Respiratory SARS-CoV-2 Panel. QIAstat-Dx Respiratory SARS-CoV-2 Panel instructions for use (Handbook). 2020. Available from: https://qiastat-dx.com/row/qiastat-dx-sars-cov-2/. Accessed: 27 Jul 2020.

8. WHO Human-Animal Interface web page. Available at: http://www.who.int/influenza/human_animal_interface/en/. Accessed: 29 Jul 2020.

9. Smith, L. Vigilant, P. A. Morin, The effects of sequence length and oligonucleotide mismatches on 5 exonuclease assay efficiency. Nucleic Acids Res. 30, e111 (2002). https://doi.org/10.1093/nar/gnf110.

10. Stadhouders, et al. The effect of primer-template mismatches on the detection and quantification of nucleic acids using the 5 ' nuclease assay. J. Mol. Diagn. 12, 109-117 (2010). https://doi.org/10.2353/jmoldx.2010.090035.

11. M. Whiley, T. P. Sloots, Sequence variation in primer targets affects the accuracy of viral quantitative PCR. J. Clin. Virol. 34, 104-107 (2005). https://doi.org/10.1016/j.jcv.2005.02.010.

12. Peñarrubia, et al. Multiple assays in a real-time RT-PCR SARS-CoV-2 panel can mitigate the risk of loss of sensitivity by new genomic variants during the COVID-19 outbreak. Int. J. Infect. Dis. 97, 225229 (2020). https://doi.org/10.1016/j.ijid.2020.06.027.

\section{Tables}

Table 1. Detection of 29 G4 swine Flu A strains by the QIAstat-Dx Respiratory SARS-CoV-2 Panel. 


\section{Flu A strain}

\begin{tabular}{|c|c|c|c|c|c|c|c|c|}
\hline Strain number ${ }^{1}$ & Strain name & & $\mathrm{Ct}$ & $\mathrm{EPF}$ & $\mathrm{Ct}$ & $\mathrm{EPF}$ & $\mathrm{Ct}$ & $\mathrm{EPF}$ \\
\hline 1 & A/swine/Anhui/0202/2018 & \#1 & 30.2 & 109,294 & $\mathrm{Neg}$ & Neg & $\mathrm{Neg}$ & Neg \\
\hline 2 & A/swine/Anhui/0203/2018 & \#1 & 30.2 & 109,294 & $\mathrm{Neg}$ & Neg & $\mathrm{Neg}$ & Neg \\
\hline 3 & A/swine/Beijing/0301/2018 & \#2 & 30.1 & 143,172 & $\mathrm{Neg}$ & Neg & $\mathrm{Neg}$ & Neg \\
\hline 4 & A/swine/Hebei/0113/2017 & \#3 & 30.3 & 67,620 & $\mathrm{Neg}$ & $\mathrm{Neg}$ & Neg & Neg \\
\hline 5 & A/swine/Hebei/0116/2017 & \#4 & 29.5 & 67,145 & $\mathrm{Neg}$ & Neg & Neg & Neg \\
\hline 6 & A/swine/Hebei/0221/2017 & \#5 & 28.5 & 45,229 & $\mathrm{Neg}$ & Neg & Neg & Neg \\
\hline 7 & A/swine/Heilongjiang/0110/2017 & \#6 & 30.3 & 71,669 & $\mathrm{Neg}$ & $\mathrm{Neg}$ & Neg & Neg \\
\hline 8 & A/swine/Heilongjiang/0140/2017 & \#7 & 32.2 & 88,997 & $\mathrm{Neg}$ & Neg & Neg & Neg \\
\hline 9 & A/swine/Heilongjiang/1214/2016 & \#6 & 30.3 & 71,669 & $\mathrm{Neg}$ & $\mathrm{Neg}$ & Neg & Neg \\
\hline 10 & A/swine/Henan/SN10/2018 & \#8 & 30.9 & 96,817 & $\mathrm{Neg}$ & Neg & Neg & Neg \\
\hline 11 & A/swine/Henan/SN11/2018 & \#8 & 30.9 & 96,817 & $\mathrm{Neg}$ & Neg & Neg & Neg \\
\hline 12 & A/swine/Henan/SN12/2018 & \#8 & 30.9 & 96,817 & $\mathrm{Neg}$ & Neg & Neg & Neg \\
\hline 13 & A/swine/Henan/SN13/2018 & \#8 & 30.9 & 96,817 & $\mathrm{Neg}$ & Neg & Neg & Neg \\
\hline 14 & A/swine/Jiangsu/J004/2018 & \#1 & 30.2 & 109,294 & $\mathrm{Neg}$ & Neg & Neg & Neg \\
\hline 15 & A/swine/Jiangsu/J005/2018 & \#1 & 30.2 & 109,294 & $\mathrm{Neg}$ & Neg & Neg & Neg \\
\hline 16 & A/swine/Jiangsu/J006/2018 & \#1 & 30.2 & 109,294 & $\mathrm{Neg}$ & Neg & $\mathrm{Neg}$ & Neg \\
\hline 17 & A/swine/Jilin/21/2016 & \#9 & 30.5 & 149,619 & $\mathrm{Neg}$ & Neg & $\mathrm{Neg}$ & Neg \\
\hline 18 & A/swine/Jilin/23/2016 & \#9 & 30.5 & 149,619 & $\mathrm{Neg}$ & Neg & Neg & Neg \\
\hline 19 & A/swine/Jilin/29/2016 & \#9 & 30.5 & 149,619 & $\mathrm{Neg}$ & Neg & Neg & Neg \\
\hline 20 & A/swine/Shandong/0334/2017 & \#10 & 29.0 & 92,841 & $\mathrm{Neg}$ & Neg & Neg & Neg \\
\hline 21 & A/swine/Shandong/0336/2017 & \#6 & 30.3 & 71,669 & $\mathrm{Neg}$ & Neg & $\mathrm{Neg}$ & Neg \\
\hline 22 & A/swine/Shandong/0337/2017 & \#6 & 30.3 & 71,669 & Neg & Neg & Neg & Neg \\
\hline 23 & A/swine/Shandong/1203/2016 & \#11 & 30.2 & 75,277 & $\mathrm{Neg}$ & Neg & Neg & Neg \\
\hline 24 & A/swine/Shandong/1207/2016 & \#6 & 30.3 & 71,669 & $\mathrm{Neg}$ & Neg & Neg & Neg \\
\hline 25 & A/swine/Shandong/16/2016 & \#9 & 30.5 & 149,619 & $\mathrm{Neg}$ & Neg & Neg & Neg \\
\hline 26 & A/swine/Shandong/36/2016 & \#9 & 30.5 & 149,619 & Neg & Neg & Neg & Neg \\
\hline 27 & A/swine/Shandong/9/2016 & \#9 & 30.5 & 149,619 & Neg & Neg & Neg & Neg \\
\hline 28 & A/swine/Shandong/JM78/2017 & \#12 & 30.3 & 117,056 & $\mathrm{Neg}$ & Neg & Neg & Neg \\
\hline 29 & A/swine/Shandong/LY142/2017 & \#13 & 30.8 & 120,913 & $\mathrm{Neg}$ & Neg & Neg & Neg \\
\hline C1 & A/H1N1/Brisbane/59/07 & $\mathrm{n} / \mathrm{a}$ & 14.9 & 190,941 & 15.0 & 81,471 & $\mathrm{Neg}$ & $\mathrm{Neg}$ \\
\hline
\end{tabular}

Page 7/8 
EPF: End-Point-Fluorescence. Neg: Negative result.

1.: Information of the 29 strains analyzed in this study were extracted from Sun et al. (1). C1 and C2 correspond to Control 1 (Seasonal human H1 subtype; Zeptometrix, Catalogue ID: 0810244) and Control 2 (H1N1pdm09 human subtype; ATCC, Catalogue ID: VR-1736). 